\title{
A Direct-Reading Two-Knife 50-Pound Balance of High Precision Suitable for State Weights and Measures Laboratories
}

\author{
H. E. Almer, H. A. Bowman, M. W. Jensen, L. B. Macurdy, ${ }^{1}$ H. S. Peiser, and B. Wasko ${ }^{2}$
}

\begin{abstract}
Performance requirements are detailed for robust fast-operating 50-pound balances precise to 1 part in about 500,000, under less than ideal weighing conditions.

Careful studies of design and configuration of large-capacity balances lead to the conclusion that the type of balance that can most easily be designed to fulfill these requirements is the one-pan, two-knife, constant-load balance.

The detailed design and construction of such a balance is described. Performance tests demonstrate that this balance conforms to the requirements. Under good operating conditions precisions of a few parts in 10,000,000 can be achieved.
\end{abstract}

\section{Introduction}

The National Bureau of Standards has been advocating systems for accurate rapid mass measurement, including highly constant mass standards ${ }^{3}$ and precise quick-weighing balances. In response to requests for cooperation, two manufacturers (the Mettler Instrument and Voland Corporations) have built balances of 50-lb and smaller capacities. Since it is the 50-lb balance that extends the high-precision direct-reading balance into a new range of capacities where no such instruments were previously available, it has been selected for the subject of this paper.

The primary purpose of this paper is to describe the design and construction of a robust but precise balance for loads up to $50 \mathrm{lb}$. The intention in section 4 below is to give all relevant details of the balance as built by the Voland Corporation so that a similar instrument can be constructed by others.

To some readers, however, details of construction may not be so interesting as the considerations on which unconventional features of configuration and design are based. To give a clear, if mostly qualitative, statement of these considerations is the purpose of section 3. Detailed features of these considerations are presented in individual specialized papers referred to in the text to the extent that these have appeared in the literature.

The stimulus to test new design schemes came from the need for large-capacity, robust, precise balances to be used for weight calibration in weights and measures offices of States. The performance requirements for balances intended for this purpose are therefore given in section 2 with a brief intro-

1 Present address Mettler Instrument Corporation, Princeton, N.J.

2 Vice President, Engineering, Voland Corporation, New Rochelle, N.Y.

3 In connection with the program, the NBS itself and two States (Hawaii and Alaska) have been supplied with highly constant mass standards of new design in series with decimal-fractional weights down to one millionth of a pound. ductory statement on responsibilities in the regulation of trade. It is not the intention of the authors, however, to suggest that the balance here described has no other potential uses. On the contrary, the need to measure large masses precisely is probably even greater in, for example, space-age industries concerned with missiles and satellites. Moreover, the new balance with its greater precision is able to provide data on mass inconstancy, evaporation rates, and adsorption or corrosion reactions within shorter test intervals.

The final short section 5 (Performance Test) describes work at the National Bureau of Standards, where not only was the adjustment carried out with considerable care, but weighing conditions are better than can be expected to prevail routinely in industry or State offices of weights and measures. It is proper that a far higher precision has been demonstrated to be obtainable with the balance than is specified in the performance requirements (sec. 2). The favorable results are indicative of the inherent soundness of the considerations on design and configuration (sec. 3). More important to many readers will be the authors' opinion that the balance as built is a robust simple stable device which a technician can learn to operate easily and quickly and on which the mass value can be read directly without intermediate observations or computations.

\section{Performance Requirements}

\subsection{Background}

The Organic Act of the National Bureau of Standards (31 Stat. 1449, as amended) includes the authorization for "The custody, maintenance, and development of the national standards of measurement, and the provision of means and methods for making measurements consistent with those standards . . . ". 
Consistency of measurement in trade and commerce throughout the Nation has been maintained through the activities of the several State weights and measures offices. The weights and measures statute of practically every State requires that the basic measurement standards of the State be submitted at least once in 10 years for evaluation by the National Bureau of Standards. The responsibility of relating these standards to the field testing equipment is assumed by the weights and measures laboratory of a State.

\subsection{The State Weights and Measures Laboratory}

The values of State mass standards are reported by the National Bureau of Standards to each of the States which in turn relates these standards to the measurements in trade and commerce within that State. Efforts of the Bureau have recently been directed toward the refinement of laboratory instruments and the simplification of their operation at the weights and measures laboratories of States.

Because the 50-lb standard is the largest mass standard normally submitted to the National Bureau of Standards by a State, even though 500-,1,000-, and 2,500 -lb test weights are in regular field use (these larger weights are compared against an accumulation of 50-lb weights), special attention has been directed to the development of a 50-lb capacity, high-precision balance that would afford excellent and repeated results with a minimum of operational technicalities and calculations.

To this time, the State laboratory has been capable of making measurements at the 50-lb range to a part in about 175,000 . Such measurements are being made on a traditional and unadorned equal-arm balance, normally through substitution comparison.

\subsection{A Direct-Reading Balance}

For a number of years there has been on the market and in laboratory use a one-pan, two-knife balance that offers high precision, direct reading, and rapid operation through a damping device. Such balances have been of comparatively small capacity, but the simplicity of design seemed to be adaptable to a capacity of $50 \mathrm{lb}$, where the advantage of eliminating calculation could increase the accuracy of the values obtained for the 500-, 1,000-, and 2,500-lb test weights, and the advantage of speed of operation could encourage more frequent calibration of these large weights.

Following studies at the Bureau, design and performance characteristics of a prototype direct-reading 50-lb balance were established as follows:

\section{a. Beam Design}

One-pan, two-knife construction with a 50-lb counterpoise fixed to the beam so all weighings would be made at the balance capacity.

\section{b. Operating Range}

To operate normally at loads of $50,30,25,20$, and
$10 \mathrm{lb}$, but with provision to operate at any load above 5 pounds and up to and including 50 pounds.

\section{c. Knife-Edge Parallelism}

Adjustable or fixed knife edges to provide highly precise parallelism for a period up to 10 years under normal use.

\section{d. Balance Weights}

Standard weights, either built-in or appropriate for hand application, to meet NBS Class S-1 requirements, such weights

(1) to be of such denominations as to approximately equal the difference between the nominal test load and $50 \mathrm{lb}$, and

(2) to be applied to that side of the balance to which the test load is applied, and, if built-in, with the weight value thus applied to be indicated on the balance dial in pounds and decimal fractions of the pound.

$$
\text { e. Indicating Element }
$$

An indicating element which, through an optical read-out with vernier, provides -70 to +70 scale divisions, readable to 0.1 division, where the 0.1 division equals $10^{-5} \mathrm{lb}$ or $4.5 \mathrm{mg}{ }^{4}$

\section{f. Scale Adjustment}

Means of adjusting the scale reading by at least $0.0005 \mathrm{lb}$.

$$
\text { g. Damping }
$$

A damping mechanism to bring about beam stability for a precise reading within 50 seconds of beam release.

\section{h. Arrestment}

An appropriate arrestment mechanism so designed as to prevent damage to the mechanism itself or to any other balance component under normal conditions of loading up to $60 \mathrm{lb}$.

\section{i. Balance Pan}

A weight-receiving balance pan so constructed as to provide at least 100 in. $^{2}$ of area for weight accommodation with at least $7 \mathrm{in}$. of clear height, so marked as to facilitate centering of the load, yet so designed as to present a minimum surface to be acted upon by air currents.

\section{j. Weight-Transfer Mechanism}

A weight-transfer mechanism to be provided by which the standard weight (or summation weight) is moved into a storage position inside the balance case and a test weight is transported from outside the balance onto the balance pan, or vice versa.

\section{k. Balance Case}

The balance to be enclosed in a case that provides adequate isolation from drafts and thermal dis-

${ }^{4}$ Compare sections 4.5 and 5 below. 
turbances and, through windows or doors, convenient access to the pan; the case to be of good appearance and of such material and design as to provide protection for at least 20 years.

\section{Limit of Extraneous Effects}

Balance shifts caused by extraneous magnetic effects, change in air density up to 2 percent, and change in relative humidity up to 10 percent to be within the limits of precision prescribed for the balance.

$$
\text { m. Precision }
$$

Standard deviation of repeated mass measurements at time of delivery to the National Bureau of Standards to be not greater than $1.6 \times 10^{-5} \mathrm{lb}$ or $7 \mathrm{mg}$.

\section{n. Readability}

Readable unit to be not greater than $1 \times 10^{-5} \mathrm{lb}$ or $4.5 \mathrm{mg}$.

o. General Design Requirement

The balance to be so constructed as to demonstrate the perfection expected of high-precision balance manufacture, and, at the same time, to be sufficiently simple of operation and rugged of construction as to give excellent results over a long period of time under conditions of use and manipulation that are less than ideal for precision weighing.

\section{Considerations on Design and Configu- ration of Large-Capacity Balances of High Precision}

\subsection{General}

The work performed by Gould $[1]^{5}$ on the perfected design of the conventional two-pan, three-knife type kilogram balance raised the state of the art to a height not previously achieved by weighing instruments intended for use at the maximum-attainable level of precision. Nevertheless, the improvement in precision obtained over previously existing instruments of this type was modest, compared with the magnitude of the effort and unsurpassed skill expended. One suspects, therefore, that the design of the two-pan, three-knife balance may be near its optimum, and that additional developmental effort would produce little improvement in precision. In the view of the present authors, it has appeared probable, at least, that any major improvement to be achieved in the near future will follow a careful study of residual weighing errors. Such a study should certainly be concerned with methods of eliminating or quantitatively correcting these errors. It is equally desirable to determine whether there is greater resistance to such errors inherent in a balance configuration different from the conventional twopan, three-knife type. Naturally, in discussions of alternative systems it is essential to make due allow-

\footnotetext{
5 Figures in brackets indicate the literature references at the end of this paper.
}

ance for new or enhanced weighing errors. Moreover, because of the possibility of unsuspected new weighing errors being introduced by a change in configuration, it is most desirable to accompany such studies with early experimental verification.

Many good studies of errors have been made and reported in the literature [2]. They are not, however, addressed to the question of configuration. In this section, therefore, some of the recent studies conducted at the National Bureau of Standards, with the primary aim of solving problems of configuration, will be described. As will be seen, these are relevant to larger-capacity balances. Moreover, their basic applicability and correctness could more easily be tested experimentally on a 50-lb balance than on a $1-\mathrm{kg}$ instrument that necessarily involves the construction of parts of great precision and delicacy.

The principal alternative to the two-pan, threeknife balance configuration is the one-pan, two-knife substitution balance [3]; its general principle of operation is well known. A fixed counterweight rigidly attached to one end of the beam balances a loaded suspension coupled to the opposite end of the beam by means of conventional end-knife assembly. The beam oscillates about a knife located between the end knife and the center of gravity of the counterweight. Ordinarily such an instrument has a group of small weights hung on the suspension. These "built-in" weights are of such magnitude that their summed moment about the center knife precisely balances the moment of the counterweight. When an unknown weight is added to the pan, the system becomes unbalanced and balance is restored by removing from the suspension an appropriate number of the "built-in" weights. The mass of "built-in" weights removed from the suspension to regain balance approximately equals that of the unknown weight. The residual mass difference is indicated on a scale calibrated directly in terms of mass.

\subsection{Errors Due to Varying Ambient Conditions}

It has been shown that variations in relative humidity [4] and temperature [5] inside balance cases may result in instrumental drift systematic with these two parameters. Housings for highprecision balances are designed to inhibit changes in temperature and humidity. Such changes thereby are made to progress slowly so that any associated motion of the beam will occur slowly. It should be noted that a precise weighing consists of the determination of a difference in load so that the absolute value of any one balance indication has no direct effect on the result [6]. The stability of the balance is a requirement only over the time taken to complete the observations constituting one weighing. A general tendency in modern instrumentation is to combat slow instrument drift by measurement systems with fast response. Existing balances of maximum attainable precision suffer from slow response. Frequencies of $10^{-2} \mathrm{c} / \mathrm{s}$ are not unusual for the oscillations of the beams of such balances. 
It has been pointed out [7] that a major portion of the experimental interval is taken up by these very low-frequency oscillations. The balance designer must therefore carefully consider how much he can increase the beam frequency of a balance without introducing new overriding weighing errors.

In an idealized knife-edge balance, oscillating about its center knife edge, $\omega=\sqrt{K / I}$, where $\omega$ is $2 \pi$ times the frequency of oscillation of the system, $I$ is its moment of inertia, and $K$ is the stiffness, which is inversely proportional to the balance sensitivity. The fast response system desired can be achieved by making $\omega$ much larger than in presently existing high-precision balances. Ideally, this should be accomplished without sacrificing sensitivity. Thus the aim is for both a large $\omega$ and a small $K$. Contrary to opinions often loosely expressed, these are not contradictory requirements. Minimizing $I$ in the design of a balance assures a maximum $\omega$ for any given stiffness. A large reduction of $I$ can be achieved by reducing the arm length. Most of the other effects of arm-length reduction, discussed below, are beneficial, but new demands arise on knife-edge parallelism. Before elaborating on these demands the discussion on ambient conditions will be concluded.

There are many methods of combating the effects of varying ambient conditions on balance operation. The housing may be insulated and thermostated; the relative humidity may be controlled; the balance itself may be constructed of nonhygroscopic materials and materials of very low coefficients of thermal expansion. All of these contrivances, and many more, have virtues and should be carefully considered by the balance builder. However, besides the importance of a fast-response measuring system, as discussed above and best achieved by a short-arm length, a most significant requirement for reducing the adverse influences from changes in ambient condition is that of rapid temperature equilibration along the beam. Any change in horizontal temperature gradient will change the effective arm ratio; to minimize such changes is another important reason for building beams with short-arm lengths. High thermal conductivity and large cross-sectional areas are also beneficial, so that the horizontal gradient will always be so small as to maintain an arm-ratio precision greater than that of the balance precision desired. It is acknowledged that this analysis differs from those of many previous balance designers who have sought high sensitivity by long, light-weight beams that are sensitive to very slight changes in temperature gradients and even mechanically deform under load. The lack of reproducibility of such deformations is a serious additional source of error in long-beam balances, and is further discussed below.

Vertical temperature differences or gradients will not be discussed in this paper, partly because they are believed to affect all beam balances similarly, and partly because they are the subject of an adjoining paper [5]. Without proof or experimental demonstration, it can be stated here that a positive verticalheight gradient (top of balance warmer) or equivalent temperature difference of the order of $10^{-3}$ to $10^{-2}$ ${ }^{\circ} \mathrm{C} / \mathrm{cm}$ is thought to be desirable for optimum performance of all balances.

\subsection{Errors Due to Knife Edges}

For a high-precision system, one attempts to develop the best quality knives. However, not even knives of highest quality are ideally perfect, and it is these slight departures from perfection that are of concern. Careful distinction should, in the authors' opinion, be made between errors from noncylindrical edges and those from curvature of the edge itself.

It should also be pointed out that in our discussion we assume that rigidly joined parts cannot move discontinuously relative to one another. For example we assume that knife edges do not move in their mountings. The assumptions can be illustrated by another example: in this discussion elastic or plastic deformation are considered, but relative movements at bolts are not. Inasmuch as real balances might be subject to engineering deficiencies permitting movements of the excluded kind, the following discussion can not be successfully applied.

The National Physical Laboratory [8] states that "in practical use the edge assumes transversely a rounded form of fairly regular curvature." Although the actual radius of this curvature may be disputed, its "regular" characteristics are universally recognized as a requirement for satisfactory balance operation. The knife behaves much like a right circular cylinder rolling on a plane surface [9], and gravimetric methods have been worked out for the measurement of the effective radius of this "regular" radius. When the knife does not remain cylindrical, it may assume an elliptical profile. The effect of elliptical knives is to change the effective arm length of the beam; in addition Corwin [10] has demonstrated that rest points calculated from observed turning points will be in error by an amount related to the amplitude of the oscillations of the beam that occur while turning points are being observed. This portion of the ellipticity error would be eliminated if the balancewere always observed at zero amplitude. This could be accomplished by damping the oscillating system, and making observations on a nearmotionless beam. The new error of starting friction on the knife is introduced by this type of operation. For all good knife-plane combinations tested, this error could not be measured and must therefore be less than 1 in $10^{-10}$ in weighing precision. The relevant tests for starting friction consist of an analysis of the deflection/time curves taken from a swinging balance with a highly sensitive read-out device [7]. Starting friction causes nonsinusoidal motion which has not been observed to the precision stated. (See also 3.6.3 below.)

Since a real knife edge is not ideally straight [10], the axis about which it rotates is not uniquely defined by the edge. It is partially defined by the longitudinal profile of the gross curvature and the load. Thus, the "regular" characteristics referred to in the foregoing paragraph may be distorted by this curvature. Damped operation will not assist here, 
but use of a constant load balance will to some extent, because the loading of a curved surface against a hard flat surface will tend to straighten out the curve by an amount dependent upon the load. In making gravimetric measurements on knife-edge radii, Hodsman and Chapell [11] found the calculated radius to be smaller under conditions of larger loads. It is believed that they were experimenting on curved knives, and that the true explanation of the phenomena was that the knives were merely straightening out under load against the flat.

So far discussion has centered on errors arising from imperfection of the knife edges themselves. At least equally important in practice are those errors arising from the imperfection of mounting of the knife edges on the balance.

In conventional balance design, the center knife in the beam points downward, and the two end knives point upward. The pan assemblies in the simplest balances are suspended by vertical rods from flats (polished on their lower side) which rest on the upward-pointing end knives. The arm length of the balance is the perpendicular distance from the center knife edge to the point on the end knife at which the suspended pan assembly load is effectively concentrated. The vertical rods connecting the pan assembly to the load flat above may be shifted from their intended vertical position by, for example, a slight error in centering when placing the load on the pan. When such a shift occurs, the effective point of support then moves along the end knife in one direction or the other. If the end knife is parallel to the center knife, no error occurs; but if it is not, motion of that point causes a change in effective arm length.

The knife-edge error just described is measured by suspending the same load, first from one end of the terminal knife and then from the other end, and noting the variation in response of the balance to the action of the load at the two different moment arms. This test is sensitive because circumstances cannot arise in which the effective point of suspension traverses more than a small fraction of the length of the end knife. Nevertheless, no practicable method is known for adjusting knives so that the parallelism error is less than the attainable sensitivity of 1 in $10^{8}$.

Thiesen [12] solved this problem by a device referred to as the Thiesen compensator which immobilizes the effective point of suspension on the end knife regardless of the verticality (or lack of it) in the suspension rods. He devised a universal coupling by placing two knife edges at right angles, one pointing upward, the other, downward. The lines of the knives mutually bisect in space; interference is avoided by removing the center sections of one knife and its associated plane. All bearing planes are mechanically constrained to coplanarity. The upward-pointing knife is the end knife on the beam, and the downward-pointing knife is attached to the suspension. The compensator, when installed, permits the suspension to swing in any horizontal direction without causing intolerable motion of the effective point of suspension on the end knife. Gould [1] and one or two other designers failed to utilize this principle because of various mechanical difficulties; however, we firmly believe that almost any one of numerous modern versions of the Thiesen compensator should be made a part of any high precision balance.

In the two-knife balance, parallelism errors are further reduced simply because the counterpoise is rigidly and permanently connected to the center knife. 'The instrumental condition of providing' three parallel lines is thus eliminated, at least to a first approximation. In this type of balance a center-line knife edge and an effective point of terminal suspension are provided. A Thiesen compensator is still required to prevent movement of the effective point of support.

\subsection{Symmetry}

If one asks a metrologist to state a few general maxims for the science and art of precise measurement, he will probably include reference to symmetry; not only for the scheme of measurement, but for the design of measuring instruments. The two-pan, three-knife balance is perhaps the most perfect example of such a symmetrical measuring instrument. It may well be that it is for this reason that scientists have not seriously questioned the conventional configuration of a balance. Indeed, the loss of symmetry inherent in the one-pan, two-knife balance would make it inferior to the two-pan, three-knife arrangement, if there did not exist methods discussed elsewhere [6] for regaining: symmetry conditions in successive readings.

In practice, the designer of two-arm, three-knife balances suffers from a serious handicap which, unfortunately, continues to divert his attention from more important features. Users demand that a balance for operation near the maximum attainable precision should give fairly accurate, direct weighings. Symmetrical distribution of masses on the two sides of the central knife edge then becomes important to the proper operation of conventional two-arm, three-knife balances. Hence, in the construction of such instruments, every effort is exerted to achieve precisely equal-arm lengths and precisely equal masses of the two suspensions, although this is not an important requirement in high-precision weighing. Even if these objectives could be achieved, they could never be maintained in practice, but the resultant error affects only direct weighings. Transposition weighing schemes are needed, although these tend to be time consuming.

Some substitution weighing procedures adapted for two-knife, one-pan balances [6] are faster than those for two-pan balances but there is a loss in sensitivity compared with two-pan transposition schemes. This loss is not serious so long as the readout sensitivity can be increased in compensation. Modern instrument-readout systems (see, for instance, [7]) permit effective sensitivities to be increased well beyond residual weighing errors. 
The symmetry problem must be discussed in one other respect. Temperature may change the rest point and sensitivity of a one-pan balance. Such a temperature effect is not easy to demonstrate and most of such effects reported to the authors could be caused by temperature gradients (see 3.2 above). A one-pan balance can, in any event, be temperature compensated. This effect, however, is not relevant here where balances are described for precise weight calibration, that is, for weighing schemes in which the effects of slow drifts are eliminated.

\subsection{Errors Associated With the Arrestor [10]}

Despite its name, the arrestor of a balance is not concerned only with arresting the motion of the beam, but has several additional functions. One of these is confusingly called "arrestment," by which the knives and flats are protected from damage during the relatively rough process of pan loading. "Arrestment" is conventionally accomplished by removing the knives from physical contact with their planes. The inverse function, called "release," is to re-engage knives and flats after pan loading. Precisely the same contact configuration that existed prior to arrestment has to be re-established. Failure to reproduce contact characteristics results in important changes in arm length and sensitivity that arise from a variety of causes, including the motion of the effective point of suspension described above, and causes gross errors in the data. Such errors are collectively referred to as the "arrestment error;" it is often the largest mechanical error associated with balance operation. The major part of the arrestment error occurs during the "release" part of the operating cycle. This operation places great demands on the arrestor, since moving parts must change positon smoothly and without vibration. Smooth operation demands that clearances between moving parts be of the "slip-fit" magnitude. Yet errors of a few microns in the placement of knives and flats during release may result in intolerably large weighing errors. Hence, these clearances no longer are in the category of "working clearance," but appear to the balance operator to be an inordinately large amount of "play." Gould [1] has demonstrated that arrestment by knife-flat separation is not a requirement for satisfactory balance operation, but he and his collaborators had great difficulty in timing the beam-swing arrestment.

In the two-pan, three-knife configuration, errors generally may occur due to imperfect-release synchronization of the two end-knife assemblies. In a configuration in two-knife balances in which there is only one end knife, the magnitude of the arrestment error is statistically reduced to $1 / \sqrt{2}$ of its usual magnitude. However, far more important is the fact that for two-knife balances it is relatively simple to design a system wherein the terminal knife as well as the center knife is kept in contact by a constant force. Pan loading then must be a careful smooth operation.
It is entirely feasible, although the need for it has not been demonstrated, to design a balance whose beam and bearings would be kept under approximately constant load throughout the entire life of the balance. Such a design would also overcome most of the problems of flexure further discussed in the following paragraphs.

\subsection{Short Arm Length}

A short-arm length has been demonstrated to be essential because of the desirability of low moment of inertia and rapid temperature equilibration along the beam (sec. 3.2). It is well to emphasize that the reduction of beam flexure is a further advantage of short beams that may be equally or even more important than the previously discussed considerations. In long-arm conventional balances, the design of the beam is such that all material not essential for the elastic support of the load must be removed. This results in filigree-type truss structures in which, first, heat flows at different rates along the various members of the structure, with the resultant establishment of temperature gradients within the beam causing distortion of the very important knife-edge adjustment (sec. 3.3); and, second, the load causes greater beam flexure. The reproducibility in the magnitude of flexure is an essential demand that is inadequately met by many conventional balances.

The beam proposed here is a short, thick, solid structure without the usual "lightening holes." Such a structure would have an equal or greater thermal capacity than conventional beams, but it would have a much lower ratio of surface area to mass. As a result, the temperature changes in such a beam would occur at a greatly reduced rate. Since the structure is solid, the establishment of nonsymmetrical distortion-causing temperature gradients is greatly reduced. These circumstances, ensure the maintenance of parallelism of the two knives (see 3.3 above). Flexure of the beam under load in short beams becomes tolerably small, and the assumption that the remaining flexure is truly reproducible and elastic becomes safe, provided a careful choice of beam material is made.

Under these circumstances the constancy of beam loadings, one of the long recognized advantages of substitution-balance configurations (often, but not exclusively, associated with one-pan balances), loses some of its importance. In other words, short beams in general will show much less variation of sensitivity with load as a result of beam flexure, an advantage that is associated with short beams as such rather than with one of the rival configurations discussed in paragraphs 3.6.1, 3.6.2, and 3.6.3 below.

Three major difficulties are encountered in an attempt to use a "very" short arm length in a conventional balance of the two-pan, three-knife configuration. Two of these are resolved in the one-pan, two-knife configuration, and the last is virtually eliminated by damping.

3.6.1. Thermal and gravimetric considerations dictate against the placement of the two balance 
pans in different horizontal planes [5]. This being the case, the overall length of the beam must exceed the diameter of one pan or else the two pans would interfere with one another. With only one pan this limitation is completely removed, but the danger of errors from differences in height between pan and counterweight caused by changing ambient conditions is still present.

3.6.2. Knife-edge adjustment becomes more difficult and critical as the arm length becomes shorter. In the conventional balance configuration, the three knives must be made parallel and the end knives must be at equal distances from the center knife. In addition, the three knives must be made coplanar, or, rather, the departure of the center knife from the plane established by the two terminal knives must be fixed and constant in magnitude. Even with a "long" beam, these requirements are difficult to achieve. In the one-pan configuration, there are only two knives and the requirements of adjustment are far more readily satisfied.

3.6.3. When excited, the pan-hanger assembly (hangdown) may swing like a pendulum about the effective point of suspension with a frequency determined by the vertical mass distribution. As the hangdown swings, the vertical force on the end knife is modulated with a frequency twice that of the hangdown. The amplitude of force modulation is proportional to the total mass of the hangdown multiplied by the square of the angular amplitude.

In passing it should be pointed out that weighing errors arising from unwanted swing of the two hangdowns do not cancel but rather add (as two independent errors) in two-pan as compared with one-pan balances. More important it is immediately clear that a most critical, feature of a balance design is a pan-release mechanism that will minimize the pan swing. For a typical balance a swing of $10^{-3}$ radians can be shown to introduce a variation in the knife-edge load of $10^{-7}$ of the total load.

It must also be considered that during a weighing the hangdown is free to pick up oscillations from the swinging beam, to an extent dependent upon the damping and resonant characteristics of the system. The amount of resonant oscillations that will be induced in the hangdown decreases as the ratio of the hangdown frequency to that of the beam decreases. Practical considerations limit the reduction in hangdown frequency, if only because of the physical height of the weights which must be accommodated. One is therefore faced with an important requirement not to reduce beam arm length so much that its frequency of swing approaches that of the hangdown. (For suppression of resonance the ratio of the frequencies should also not be that of simple integers.)

Discussion with several older balance craftsmen has taught the authors that this limitation to shortening the beam has been understood in the past. However, it was always assumed that balance readings must be taken "on the fly," when the hangdown oscillations produce an uncertainty of turning points, the magnitudes of which are not easily recognized, unless very low beam frequencies are used.
In section 3.3 above, the conclusion was reached that it is advantageous to weigh with a damped beam. An additional major advantage gained from damping: is that the readings taken with the beam at the equilibrium rest point automatically eliminate, by averaging, any residual error due to swing of the hangdown. Incidentally, optical magnification of balance deflection [7] at the rest point clearly shows that oscillations due to pan swing are still observable, and that a good knife edge does not "stick" because of starting friction which must be less than about $\pm 10^{-10}$ of the load force. The damping system used need not be further discussed here as simple trials have shown that for large-capacity balances, nearly the optimum damping (about 0.7 critical) can be achieved by several devices without otherwise disturbing the balance appreciably. Damping systems, however, do become more difficult in smaller balances of high precision, and should receive further study.

\subsection{The Sail Effect}

Even in a well-designed balance case under operating conditions in which the vertical temperature gradient is carefully controlled, there will be thermal gradients in the contained air which will set up convective air currents. The resulting adverse effects of convection can be reduced by keeping to a minimum areas on beam and pan assemblies exposed to possible air currents.

These air currents are often rotary within the balance case so that their effect on two-pan balances may be additive (i.e., "up" on one pan and "down" on the other). With only one pan on which the current can act, the effect is significantly reduced. If this one pan is centrally placed in the balance case so that it hangs in relatively quiescent air, there is an even greater reduction of this "sail effect" error.

\subsection{Conclusion}

On the basis of the above discussion it can be concluded that a simple robust one-pan, two-knife balance of high precision can be built, provided it is a fast response, damped, short-arm instrument. The beam should have a large cross-sectional area and be made of mechanically strong material of high thermal conductivity. A Thiesen compensator in the suspension, a good pan-release mechanism, and a gently operating weight-transfer system would also be needed. High quality knife edges rigidly mounted in the beam and many other features of highprecision balances, such as a good optical read-out system, would require great care. The demand for a short arm led to the suggestion of a horizontal cylindrical counterweight at right-angles to and bisected by the beam plane. In this way, an effective arm ratio of unity could be achieved, integral fabrication with the beam seemed feasible, and the moment of inertia for any given restoring moment required of the counterweight could be kept small. 


\section{Design and Construction}

\subsection{Introduction and General Comments}

The unique requirements of exceptional precision coupled with previously unattained stability, ruggedness, and suitability for use by personnel having relatively little training, have been met by novel design based on the analysis outlined above and considerable experimentation during development. The one-pan, two-knife balance stands in its case with its beam perpendicular to the front panel. It can be operated by means of three major controls. (See fig. 1.) The first is the control lever which operates the turntable (fig. 1a) for weight transfer and the beam arrestment. The second rotates the turntable (fig. 1b). The third is the pan-arrest lever (fig. 1c). All these controls are at the front of the case and readily accessible to the operator. The optical image screen presenting the balance reading and the associated microvernier also appear in front of the balance case.

Materials were chosen to provide long life and to require minimum maintenance. All shafts rotate in either porous bronze bearings or in ball bearings.

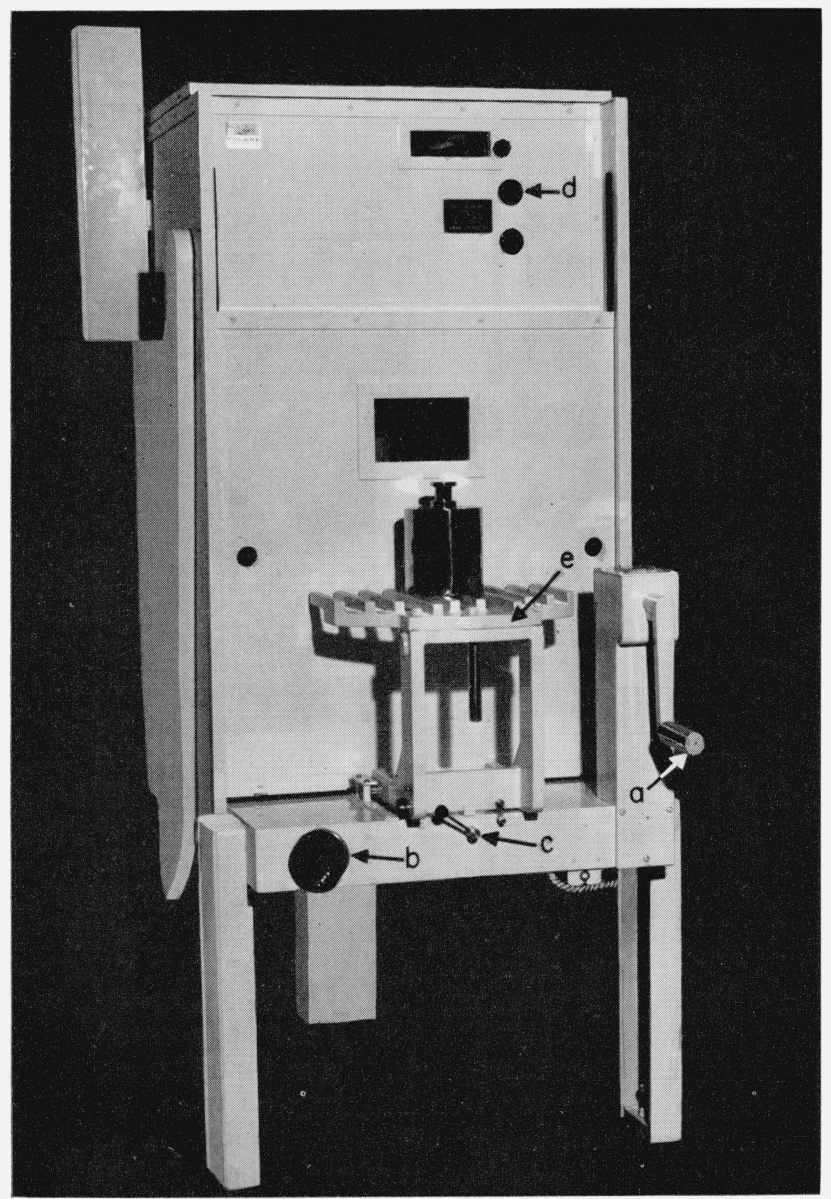

Figure 1. General view of 50-lb balance.
The knife edges are of Tantung $\mathrm{G}$ and the bearing's of Brazilian agate. This combination seems to provide excellent life. The beam material is manganese bronze to provide a combination of adequate strength, rigidity and conductivity, and to give a counterweight density of approximately $7.9 \mathrm{~g} / \mathrm{cm}^{3}$ to match the stainless steel weights that are most likely to be used.

\subsection{The Beam With Fixed Counterweight}

The beam is short (arm length, $2 \frac{3 / 4}{4}$.) and of low moment of inertia with an effective arm ratio of about unity (fig. 2). The fixed cylindrical counterweight (fig. 2a) is cast as an integral part of the beam. The axis of the counterweight is horizontal and perpendicularly bisected by the beam plane. ${ }^{6}$ The beam and counterweight weigh about 70 pounds. In manufacture, the counterweight is machined to a true cylinder within \pm 0.0005 in. to serve as a reference for further machining.

The effects of alinement errors and dimensional instability of the knife edges increase with decreasing arm length (see sec. 2.3). A novel and improved knife-mounting system has therefore been found essential. The beam is prepared for the center knife by a $90^{\circ}$ inverted groove with horizontal axis perpendicularly bisected by the beam plane. The sides of the $V$-grooves which serve as the knifelocating surfaces are inclined by about $45^{\circ}$ to the beam axis (fig. 2b). A clearance slot is machined into the top of the groove. The knife itself is a rectangular bar having a length of 2 in. and square cross section of side $1 / 2$ in. Opposite faces are lapped parallel and adjacent faces are approximately at $90^{\circ}$, opposite angles being equal. One pair of angles is matched with the groove angle of the beam so that the intersections of the four rectangular optically flat faces form two interchangeable knife edges. Clamps (fig. 2c) prevent sliding only; screw adjustments are not needed. In this way stresses due to screws and other beam strains can be avoided. The short beam can be built rigidly enough to make groove spread under load negligible. The load knife is shorter (11/4 in.), but otherwise it and its groove are of a design identical with that for the center knife.

Screw trimming weights (fig. 2d) for balance and sensitivity are conventional as is a 100 in. $^{2}$ air damper (fig. 2e) 13 in. from the center knife operating in a $5 \times 20$ in. tray with vertical walls.

\subsection{The Beam Arrestment}

Whereas the load-supporting terminal knife edge is not separated from its flat during a weighing series, a carefully designed arrestment system for the center knife has been provided. With it the usual arrestment errors, arising to a substantial part at the terminal knife edge (see sec. 3), are at least reduced.

6 Vertical plane containing the beam axis normal to knife edges. 


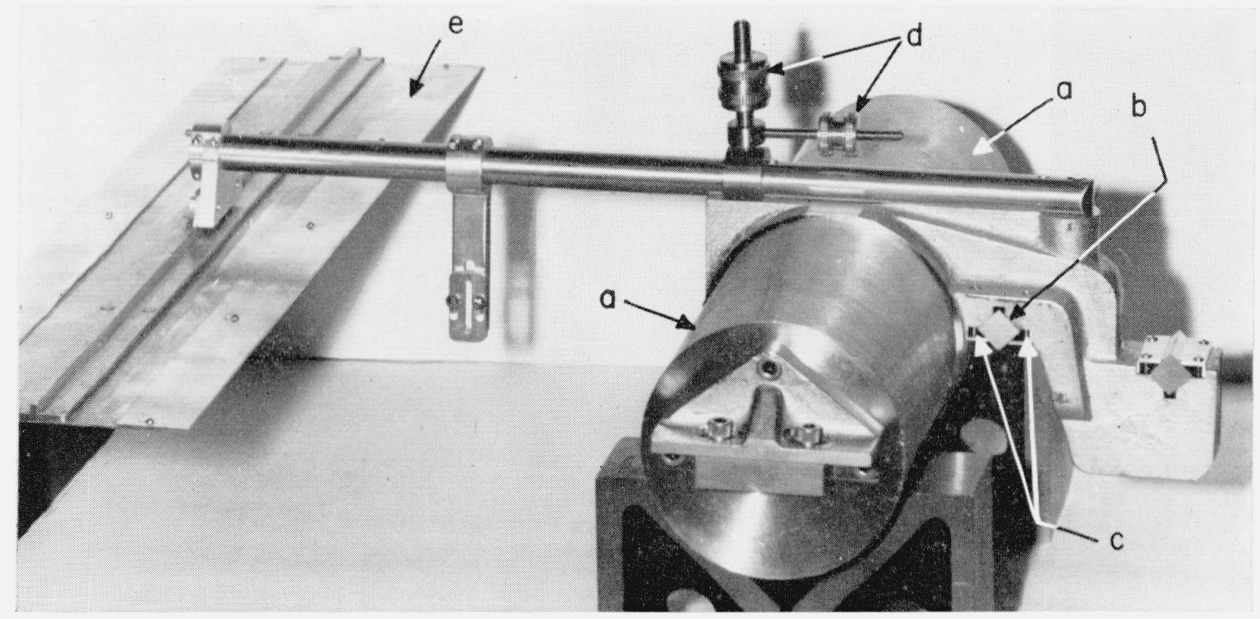

Figure 2. Weighbeam assembly.

The principal, rigidly designed, arrestment arm (fig. 3) is cam operated and pivoted about a horizontal shaft fixed to the massive aluminum bed plate. This shaft is located $15 \frac{1}{2} \mathrm{in}$. from the terminal knife. The arrestment arm carries three vertically upward pointing fingers (inverted cup, V groove, and a plane) which engage three points fixed to the upper plate of the hangdown (figs. 3a and 4a). The pan arrestment mechanism (see below) serves to raise the pan end of the balance gently until two upward-pointing fingers (fig. $3 b$ ) rigidly fixed to the bed plate engage a downward-pointing slot and cup (fig. 3c) on the ends of the counterweight. The principal beam arrestment arm then slightly raises the hangdown so that the beam pivots about the two points on the counterweight, disengaging the center knife from its flat. The satisfactory operation of this arrestment depends on adequate parallelism of the line joining the fixed points with the center knive. The cup and slot therefore have a floating adjustment carried out by the manufacturer to \pm 0.0001 in. to minimize the arrestment error. Instability of temperature dependence of this adjustment has not been detected.

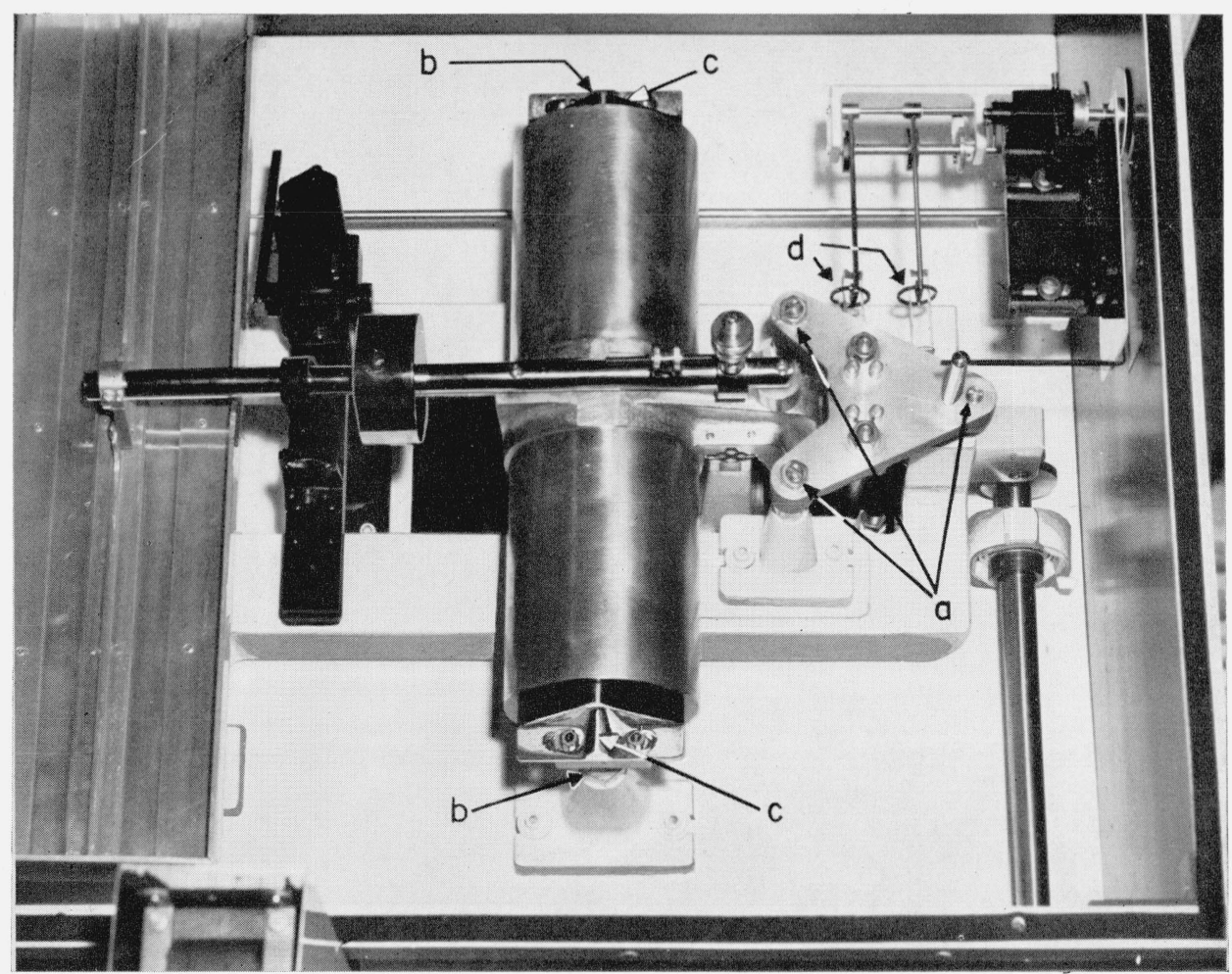

FIGURE 3. Bedplate assembly with arrestment, beam, optical system, suspension, and calibration mechanism. 


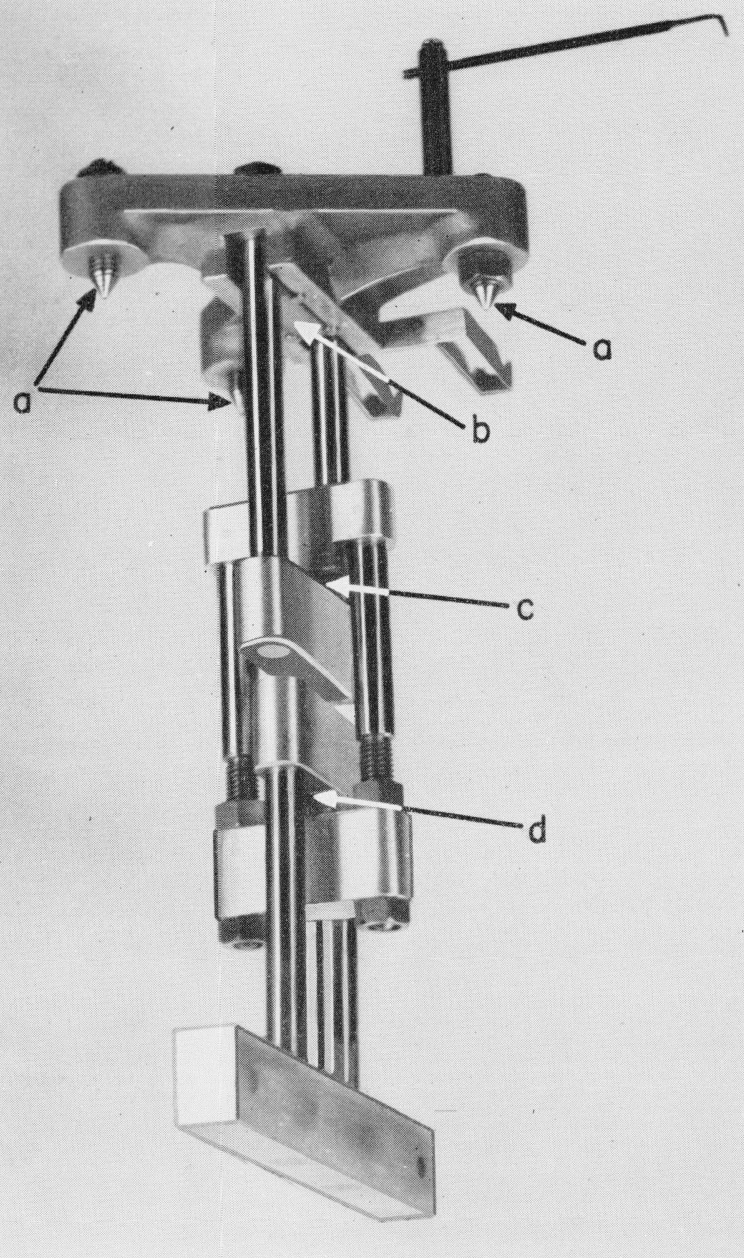

Figure 4. Hangdown assembly.

\subsection{The Hangdown and Pan Arrest}

The hangdown and pan are shown in fioures 4 and 5a. The weight-bearing plane is 25 in. below the terminal flat (fig. 4b). The aluminum support rods $(3 / 8 \times 1$ in. section) are symmetrically placed on either side of the vertical center line under the terminal knife and in a plane perpendicular to the beam plane.

As discussed in section 3.3, compensation for misplacement of the weight on the pan perpendicular to the knife plane is required. Ideally, the compensating knife edge should be coplanar with the terminal knife edge. In practice, a vertical displacement can be tolerated if it is small compared with the distance from the terminal knife edge to the center of gravity of the loaded pan. The additional knife edge (fig. 4c) in this balance is actually 3 in. below the load-bearing knife.

Although the terminal knife does not leave its bearing on arrestment, a mechanism for ensuring that the center of gravity of the hangdown with load is vertically below the terminal knife edge and for damping out pan swing is a critical design feature. On final release no horizontal force component must be allowed to be exerted upon the pan. These requirements are achieved by a $(3 / 16 \mathrm{in}$. diam) button (fig. 6a) of stainless steel acting on the underside of the pan; the area of contact is polished. The button is fixed to a horizontal arm (fig. $6 \mathrm{~b}$ ) pivoting on horizontal knife edges $9 \frac{1}{4}$ in. from the button. At the opposite end of the frame are

(1) an adjustment counterweight to determine the force applied by the button, and

(2) a hydraulic dashpot that controls the upward vertical movement of the button.

The pan arrest operates in the following manner:

(1) In the arrested position the counterweight presses the pan-arrest button against the bottom of the pan with a force just sufficient to permit the pan to damp to zero velocity in a position very near to gravitational alinement of the pan assembly.

(2) When the beam is released, the pan-arrest button follows the vertical motion of the pan, but still provides sufficient force to dampen out the horizontal motion of the pan.

(3) The pan-arrest button is pulled away from the pan by a cam-operated finger.

During the above three steps the hydraulic dashpot is, for practical purposes, not part of the system.

The first step in arresting is to permit the button slowly to contact the bottom of the pan. Provided the beam is nearly balanced, the force of the panarrest button will push the pan vertically at a rate controlled by the dashpot. The beam will tilt back to its limiting position with the cup and slot on the counterweight in position against the fixed fingers on the bed plate. With this device there is no tendency to jar the bearings during arrestment.

An additional device is included to eliminate the possibility of motion of the terminal knife relative to its flat. It has been found that even relatively small disturbances to the load pan, particularly if in the form of a torque around a vertical axis running through the center of the knife, can introduce variability in readings. For this reason, the pan hanger is supported on a pivot (fig. $4 \mathrm{~d}$ ) with freedom to rotate around a vertical axis. In order to prevent rotation of the hanger about a vertical axis during loading and unloading of the pan, locating pins operated by the arrestment mechanism are inserted beneath the hanger. This device will be discussed below (in sec. 4.6) in connection with a procedure for centering the load on the hanger pan.

The load hanger is designed to offer a minimum surface to the movement of air within the weighing chamber. The load pan consists of a slotted plate in the form of a fork open towards the rear of the balance case. Below and at the two sides of the pan there is a rack on which counterweights can be placed manually in a symmetric array. Adjustable counterweights on the hanger permit it to be leveled with respect to the load-transposing mechanism described below (sec. 4.6). 


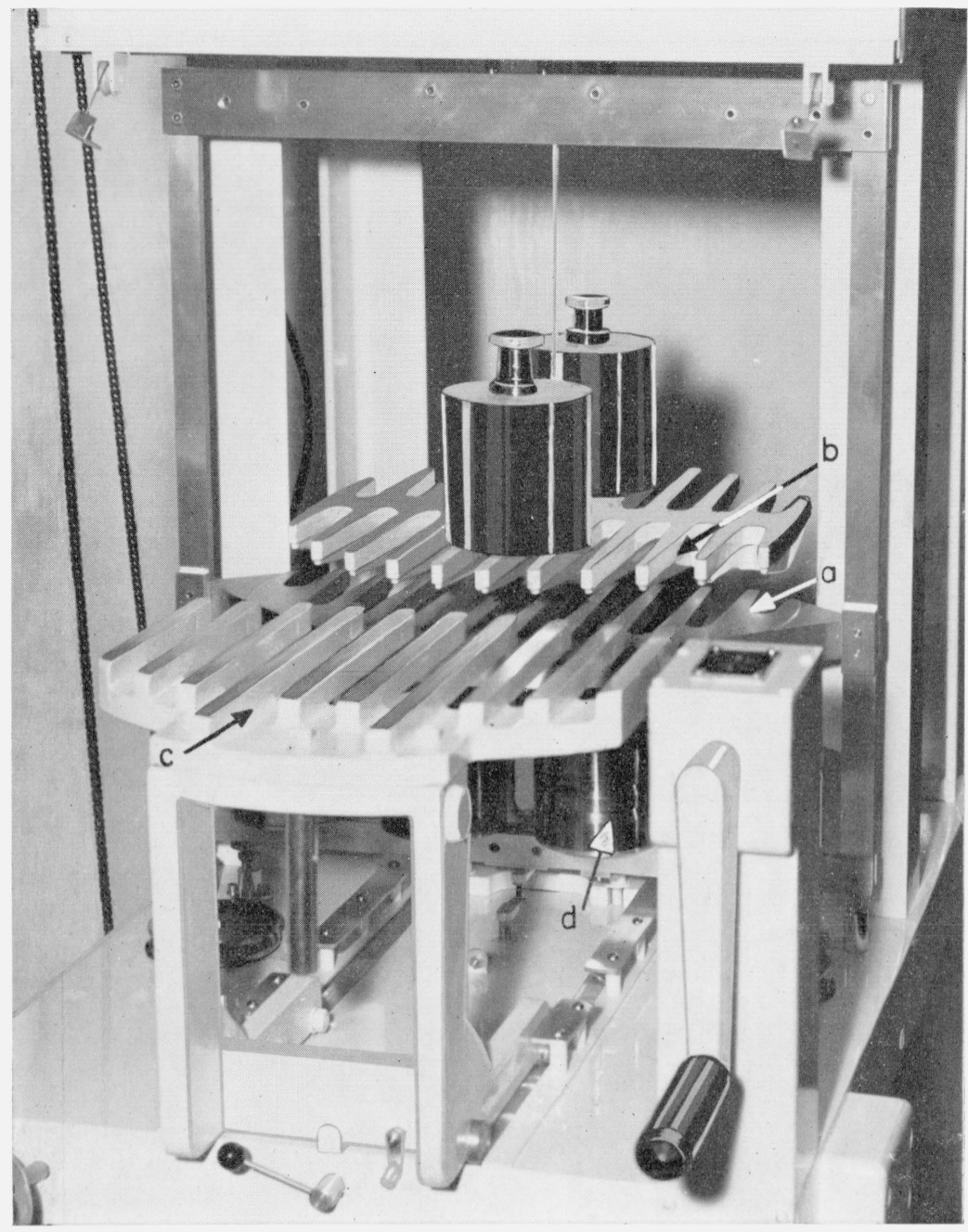

FIGURE 5. Weight loading and transposing mechanism with truck, slotted pan, and elevating turntable.

\subsection{The Read-Out}

A graticule mounted on the end of the beam is graduated with $140(+70$ to -70$)$ radial lines (9 in. radius) equally spaced at $1_{1 / 2}^{1 / 2}$ angular minutes per line. It is fixed in the beam plane 9 in. from the center knife. A conventional projection system brings the image of the graticule to a screen at the front of the case. A cam-operated microvernier ${ }^{7}$ permits ready estimation of the graduated scale to within $1 / 100$ of a division from wide vernier marks representing $1 / 20$ of a scale division. This is 10 times that required in the original specification, but the performance of the balance is such that the additional readability could be useful for potential uses of the balance.

In the design of the optical system, all heatproducing elements are placed outside and above the top of the case to minimize thermal effects.

${ }^{7}$ This readout was incorporated as a result of the performance test (see sec. 5).
The residual heat flow into the balance helps to establish the positive gradient discussed in section 3.2. In addition the entire beam, arrestment and optical systems are mounted on the heavy bed plate which provides a thermal sink and reduces vertical movement of the air within the weighing chamber.

Two ring weights (fig. 3d) that have a mass corresponding to 50 scale divisions are supplied. The rings are so operated by a dial (figs. 1d and 3d) that with this dial at "zero," one of the rings is on the hanger. With exactly 50 pounds on the pan and the ring in place, the beam should balance with the scale indication at zero. When the control knob is set to "plus," the second ring is added to the hanger. It causes the balance to indicate +50 divisions provided it is properly adjusted. When the control knob is set to "minus," both rings are removed and the indication should be -50 divisions. If the balance is not properly calibrated (or if the ring weights need to be cleaned), the indication will be incorrect. 


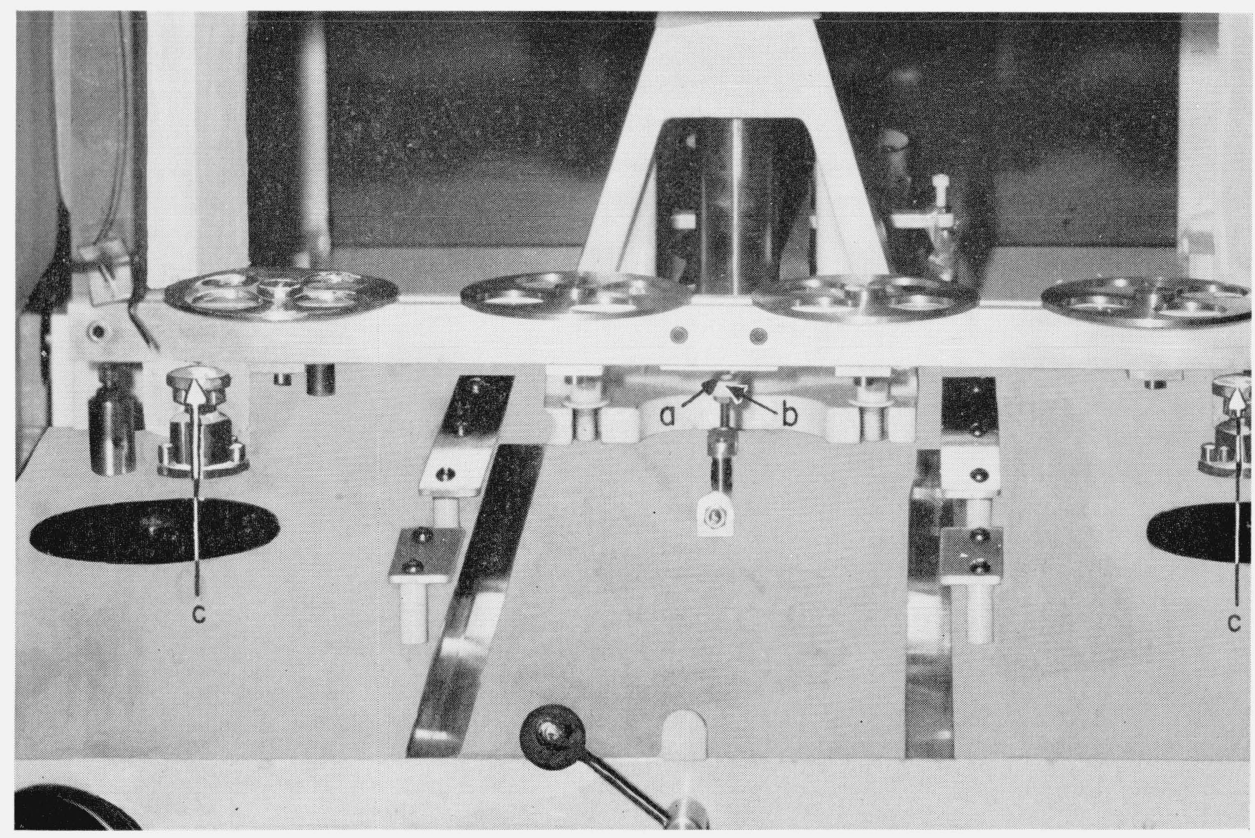

Figure 6. Pan arrestment and load centering mechanism.

A small nut on the beam can be adjusted vertically to bring the beam to the proper sensitivity.

A wide-range zero adjustment is also provided. For this adjustment the image of the projection scale can be moved by rotation of a flat mirror in the optical path. The balance can thereby be set to the accepted mass correction when weighing a standard weight. When weighing an unknown weight, no further arithmetic correction for the balance indication is necessary [3].

\subsection{Weights and Weight Loading}

The standard weight and unknown weight (or group of weights which constitute the unknown) are stored on a two-position turntable (fig. 5b) which can be rotated through $180^{\circ}$. The turntable is made in the form of a slotted plate which interlaces the pan of the balance and is located behind the pan and within the weighing chamber. It has vertical motion so that its weight-bearing plane can be raised to clear the top of the balance pan or lowered to a position below the weight-bearing plane of the pan. In the raised position, the turntable can be rotated to present either of two weights to the pan and is provided with two controls, one for elevation and one for rotation. Stops are incorporated for accurate positioning. Since the elevating mechanism is required to support a load of $100 \mathrm{lb}$ consisting of a $50-\mathrm{lb}$ test weight and a 50-lb standard, it has been decided that a multiple-turn handle is needed. This system can be operated without undue exertion.

Because of the difficulty of handling, the weights are introduced into the weighing chamber by means of a truck (figs. 1e and 5c) normally located outside the balance cabinet and capable of overriding the pan. Markings are provided on the truck to center the weights. However, since it is difficult to center the weights exactly, the following procedure is used ${ }^{8}$ for the centering of the weights on the pan.

1. The turntable is lowered to a position just below the top of the truck. This position is indicated on a slide-rule dial connected to the operating handle.

2. The truck (fig. 1e) is moved into position just above the pan. The turntable is elevated until the weight is lifted off the truck, which is then removed.

3. The turntable is lowered transferring the weight to the pan. Until this time, two locating pins (fig. 6c) have been engaged in the bottom of the pan frame to prevent movement. These pins are automatically retracted just before the weight is transferred to the pan. After the weight is transferred to the pan, the pan swings, controlled by the panarrest button, into a position in which the center of gravity of the assembly is below the knife system.

4. The turntable is raised again until the weight is just lifted off the pan. The locating pins do not re-enter the pan frame so it is free to swing back to its normal vertical position.

5. The weight is so raised from and lowered onto the pan several times. At each successive stage the hanger assembly is brought nearer to gravitational alinement. After approximately three cycles there is no longer discernible movement of the pan during the transfer. The slide-rule dial indicates the correct position of the turntable so that it is not necessary actually to observe the transfer of the weight,

8 The procedure includes changes in weight arrestment suggested as a result of preliminary tests (see sec. 5 ). 
and the operation can be accomplished with the cabinet door closed. The weights can then be stored on the turntable without disturbing this adjustment.

Once the weight has been centered the turntable can be lowered completely out of the way. Continued rotation of the arrestment lever releases the beam as the elevator reaches its lowest position. The only further operation required to make a weighing is to operate the pan-release mechanism. In particular it is not necessary again to open the balance case doors, since the centering of the weights on the turntable remains undisturbed on careful rotation.

All the weight-transfer mechanisms are fastened to a heavy aluminum plate which is welded to an aluminum frame for rigid support. The plate also helps to provide thermal stability.

Since the $50-\mathrm{lb}$ balance is intended to calibrate weights of a limited number of nominal mass values, individual tare weights are supplied to bring the balance to its full capacity of $50 \mathrm{lb}$. The cylindrically symmetric tare weights are made of stainless steel and shaped so that they can be stacked symmetrically on either side of the hanger pan (fig. 5d). The weights are arranged so that any value from zero to $50 \mathrm{lb}$ in 5 -lb steps can be calibrated. For ease of handling, no weight is greater than $10 \mathrm{lb}$. For symmetry, the smallest weight must be $2.5 \mathrm{lb}$.

\subsection{The Balance Case}

So that thermal effects will be minimized, the 42 in. $\times 25$ in. $\times 26$ in. cabinet has been designed to have both good insulating characteristics and low heat capacity. The cabinet panels are of doublewall construction with thin aluminum plates over a skeleton plywood support of $1 / 2$ in. thickness. The space between the plates is such that the air trapped between them does not tend to circulate. The outer surfaces of the panels are painted a glossy white to reflect radiant heat. The inside is painted dull black to approximate to blackbody conditions. The panels are bolted to the heavy supporting members of the balance, and the counterbalanced front panel can be raised and lowered on slides. A small viewing window is provided.

\section{Performance Test}

Results of the tests on the 50-lb one-pan, two knife, substitution balance given below demonstrate that a high-precision balance can be designed and built according to the foregoing principles and specifications.

Because this balance is of untried design, some preliminary tests and weighings were made to help the authors to become familiar with the balance before proceeding with the evaluation tests. During this preliminary work, the need for some means of centering the load on the pan became apparent. This requirement was met by changing the arrestment cycle so that the load was transferred from the turntable to the pan before the load on the suspension was transferred from the arrestment mechanism to the terminal knife of the balance beam.
This change made it possible to center the load on the pan by repeatedly putting the load on the pan and removing the load from the pan before the balance was released. When this change of the arrestment cycle had been made and the balance carefully adjusted, the performance test was made. The performance of the balance during this test exceeded the requirements specified in the development contract.

Because balances of this type will not always be used under the best attainable conditions, the tests were made under both good and poor weighing conditions. The balance was first tested under the conditions normally existing in the mass laboratory of the National Bureau of Standards. The second part of the test was made under simulated adverse conditions. The unfavorable conditions were created by heating the base of the balance radiantly and directing a current of hot air toward the balance. The performance test consisted of several series of weighings; each weighing measured the difference in the mass value of two weights having the same nominal value. The same two weights were used throughout a given series. The agreement of the results of the weighings in a given series was a measure of the performance of the balance. This measure was expressed as the standard deviation of a single weighing.

During the first part of the test the precision of weighing as measured by the standard deviation of a single weighing was 3 to $5 \times 10^{-6} \mathrm{lb}$, or about 1 part in 10 million. The standard deviation of a single weighing during the second part of the test was about $15 \times 10^{-6} \mathrm{lb}$, or about 3 parts in 10 million. On the basis of this test, the maximum weighing error that can be expected under adverse conditions is about one part in a million.

During the performance test, it was found that a large part of the imprecision of the balance could be ascribed to arrestment errors. A number of tests were made to determine if any single part of the balance mechanism was responsible for a significantly large part of these errors, but the results of these tests were inconclusive.

The tests demonstrated not only the balance's capabilities, but also the desirability of greater resolution in the read-out than the $1 \times 10^{-5} \mathrm{lb}$ of the original reading device and the need for a pan large enough to accommodate groups of weights with a total nominal value equal to the capacity of the balance. Therefore, two further modifications were made: The reading device was replaced by one readable to $1 \times 10^{-6} \mathrm{lb}$; and the pan, turntable, and loading truck were replaced by similar parts large enough to accommodate groups of weights having a total mass value equal to the capacity of the balance.

After these modifications had been made the balance was placed in service as a calibration balance in the Bureau's mass laboratory. In this service, the precision of the balance as measured by the standard deviation of a single weighing is about 1 part in 10 million. 
It is a pleasure and a privilege to acknowledge most gratefully the collaboration and advice from many experts at the National Bureau of Standards, at other research institutes, and balance manufacturing firms. It is difficult to choose just a few individuals for special mention, but Miss E. M. Clinton has carried out independent confirmatory investigations freely communicated to the authors. Professor A. H. Corwin by personal interest and as member of the Advisory Panel at the NBS Metrology Division, has often discussed this work most helpfully with the authors. Mrs. M. W. Jones is hereby thanked most cordially for her technical and experienced support of this and other researches in the Mass and Volume Section of the Bureau. Last but not least the authors express their appreciation for the kind background help always available from the NBS Editorial Section.

\section{References}

[1] F. A. Gould, Proc. Phys. Soc. B62, 817 (1949).

[2] A. H. Corwin, Weighing techniques of organic chemistry, Vol. I, Physical Methods of Organic Chem., 3d edition, pt. I, Ch. III, Interscience Publishers, New York (1949).

[3] H.S. Peiser, State mass standards, Rept. 45 Nat. Conf. on Weights \& Measures, 45 (1960).

4] H. E. Almer, J. Research NBS, 64C, 281 (1960).

[5] L. B. Macurdy, J. Research NBS, 68C, 135-40 (1964).

[6] H. E. Almer, L. B. Macurdy, H. S. Peiser, and E. A. Weck, J. Research NBS, 66C, 33 (1962).

[7] H. A. Bowman and L. B. Macurdy, J. Research NBS, 63C, 91 (1959).

[8] NPL Staff; Balances, weights, and precise laboratory weighing; NPL Notes on Applied Science No. 7, Her Majesty's Stationery Office (1954).

[9] G. Guglielmo, Concerning two methods of determining the radius of curvature of the surface of the knife edges of balances and pendulums, Atti reale accad. lincei, Series 5, 11, 2d half-year (1902).

[10] H. A. Bowman and H. E. Almer, J. Research NBS 6\%C, 227 (1963).

[11] G. F. Hodsman and F. A. Chappel, J. Sci. Instr. 29, 10 Oct (1952).

(Paper 68C3-159)
[12] M. Thiesen, Trav. et mem. bureau international des poids et meas., 5, pt 2 (1886). 